\title{
Bemerkungen zu den Gedichten vom Knechte Jahwes.
}

Von Prof. Dr. Franz Praetorius in Breslau.

Im wesentlichen sind die folgenden Bemerkungen textkritischer Art. Nur selten habe ich Veranlassung gehabt, dem Sinnesinhalt der Gedichte näherzutreten. Hinsichtlich der Grundauffassung derselben trete ich denjenigen bei, die in dem Knechte eine Einzelperson sehen.

\section{I.}

Es fällt auf, daß Jes 42 I a und v. 2 umgekehrte Pentameter sind $(2+3)$. Da dieser sonst recht seltene Vers hier in kurzem $Z$ wischenraum zweimal vorkommt, so trage ich Bedenken zu verändern, wie LEY möchte (Theol. Stud. u. Kritiken 1899, 197). Vielmehr nehme ich an, daß zunächst v. I uns unversehrt überliefert worden ist und aus einem umgekehrten Pentameter besteht ( $\mathrm{r}$ a), dem ein gewöhnlicher Doppeltrimeter folgt ( $\mathrm{I}$ b). Auch in $\mathbf{v} .2$ sehe ich sodann einen unversehrten umgekehrten Pentameter.

Aber betreffs des Folgenden bin ich nicht sicher. Ich halte es nicht für unmöglich, dal der ganze dritte Vers und die ersten vier Worte des vierten Verses zu streichen sind. Dann würde dem umgekehrten Pentameter von v. 2 auch hier wieder ein gewöhnlicher Doppel-



Die übliche Auslegung von v. 3 a scheint nicht die einzig mögliche zu sein. Es liegt mir fern, die folgende Erklärung als sicher anzusprechen; aber möglich scheint sie mir zu sein, und demzufolge wird man auch die bisherige Erklärung nicht mehr als ganz sicher annehmen können.

Ein geknicktes Rohr zerbrechen und einen verlöschenden Docht auslöschen sehen wie sprichwörtliche Redensarten aus. (Vgl. SELLIN, Rätsel des deuterojes. Buches 103.) Es wäre denkbar, daß diese Redensarten besagen sollen: Mit einem Gegenstande, mit einer Hand- 
lung nicht viel Mühe haben. Und da beide Sprichwörter hier negiert sind, so würde der Sinn sein: Er wird tüchtig zu tun haben, bis er auf Erden das Recht festsetzt. Man könnte aber auch annehmen, daß der Sinn der sei: Ein schon geknicktes Rohr überflüssigerweise nochmal zerbrechen, einen schon von selbst verlöschenden Docht überflüssigerweise noch besonders auslöschen. Es würde dann ungefähr unserer Redensart entsprechen „offene Türen einrennen“, d. h. sich den Anschein großer Tätigkeit geben, während im Grunde alles ganz leicht geht. Erwägt man nun, was in v. 2 steht, so scheint es mir einigermaßen wahrscheinlich, daß der Sinn der hebräischen Sprichwörter ähnlich dem der angeführten deutschen Redensart sein könnte: „Er wird nicht laut schreien und auf der Gasse nicht seine Stimme erschallen lassen; er wird von seiner Tätigkeit nicht viel Aufhebens machen, bis -“. Vielleicht stand v. 3 a als erläuterndes, paralleles Sprichwort einst nur am Rande. Seine in dem gewöhnlichsten Metrum laufende Form ist kein Beweis für das Gegenteil.

Wie dem auch sei, jedenfalls ist v. 3 a glossiert worden. Was der Glossator sich gedacht hat, der zu v. 3 a die vier Worte zugesetzt hat, die jetzt am Anfang von v. 4 stehen, wird mit Sicherheit nicht auszumachen sein; umsoweniger als ihr Wortlaut nicht ganz feststeht, und Sept. etwas abweicht. Am wahrscheinlichsten ist mir, daß der Glossator die Deutung hat abweisen wollen, der Gottesknecht sei das geknickte Rohr, der verlöschende Docht. Deshalb schrieb er: „Er wird nicht verlöschen und wird nicht geknickt werden."

$\mathrm{Dab}$ v. 3 b Glosse zu v. I $\mathrm{b} \beta$ ist, scheint mir sicher, wie immer man über לאמת denken mag.

\section{II.}

Das zweite Gedicht, $49 \mathrm{I}-6$, enthält zu Anfang in v. I und 2 je zwei Doppeltrimeter, die völlig unversehrt zu sein scheinen, und deren allgemeiner Sinn klar ist. $\mathrm{Er}$ steht insofern in Übereinstimmung mit $42 \mathrm{x}-4$, als hier wie dort die Rede ist von einer von Gott erwählten Person, deren. Tätigkeit bis zu einem gewissen Zeitpunkte zurückgehalten wird.

Es ist mir nicht klar, wie man in v. 3 ḥְִ? grade des Metrums wegen hat streichen wollen. M. E. verlangt das Metrum gebieterisch seine Beibehaltung:

wayyōmär lī 'abdí attá yisraél ašer bák etpaár.

Wollte man tọ streichen, so hätten wir in v. 3 einen vereinzelten 
Pentameter unter den vorangehenden und folgenden Doppeltrimetern. Die äußere Form gebietet also, das Wort als zum ursprünglichen Text gehörig beizubehalten und sich mit seinem Sinne auseinanderzusetzen.

Der Dichter hat mit לִיר: m. E. an dieser Stelle eine einzelne Person im Auge gehabt, aber doch wohl eine das ganze Volk deckende. Ungefähr so wie wir von einer Germania sprechen: Wir haben dabei eine einzelne Gestalt vor Augen, aber sie bedeutet uns das ganze Volk. So steht in der vorliegenden Dichtung die Einzelgestalt Israel dem Volke Jakob gegenüber und deckt sich in der Wirklichkeit doch mit ihm. $\mathrm{Ob}$ diese Einzelgestalt Israel ursprünglich einen anderen, historischen Namen geführt und erst später vom Dichter mit dem Volksnamen Israel belegt, dem Volke angeglichen worden ist -, für diese Frage kann ich neue Gesichtspunkte nicht beibringen. Es scheint aber, $\mathrm{dab}$ die Unklarheit der Dichtung in diesem Punkte, wie sie nun tatsächlich einmal vorliegt, schon früh zu Mißverständnissen geführt hat, von denen der uns überlieferte Text Zeugnis ablegt.

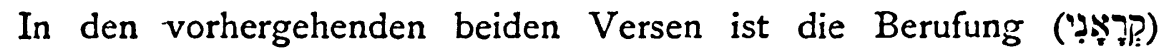

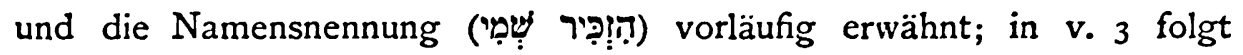
das Nähere: „Und er (Jahwe) sprach zu mir: Mein Knecht bist du, o Israel!“. Ebenso wird der in v. 2 nur vorläufig angedeuteten späteren Tätigkeit des zum Knecht Jahwes Berufenen in v. 3 eine besondere Färbung gegeben, „an dem ich mich verherrlichen will“. Dieser Relativ-

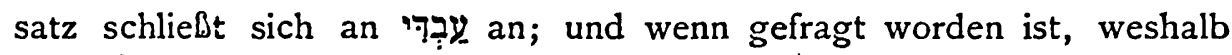

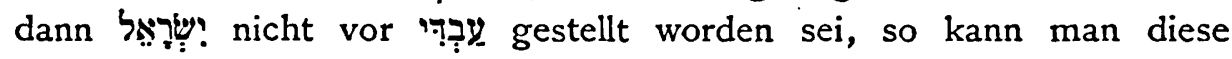
Frage mit dem Hinweis auf das Metrum beantworten - ganz abgesehen davon, daß auch ohne den $Z$ wang des Metrums die Trennung des Relativsatzes von seinem Substantiv durch einen Vokativ sehr wohl denkbar wäre.

V. 4 enthält wieder zwei Doppeltrimeter, von denen der erstere leicht überfüllt ist. Ob ṣִ $\mathrm{zu}$ streichen, lasse ich dahingestellt, denn vielleicht wurde die Wortgruppe im Gedichte zu wanimárti reduziert; sicher aber ist sich auf die babylonische Gefangenschaft zu beziehen. Israel hat trotz allem die Hoffnung auf Jahwes Vergeltung nicht aufgegeben. Und diese wird ihm in der Folge in unerwarteter Fülle zuteil.

In v. 5 beschließt Jahwe zunächst, daß Israel als Knecht Jahwes (das Volk) Jakob zu ihm (nach Jerusalem) zurückführen soll. Aber der Vers enthält schon einen Vorausblick auf noch Größeres: „und ich bin wertgehalten worden vor Jahwe." 
Der erste Doppeltrimeter des masoretischen Verses geht bis und ist $\mathrm{m}$. E. unversehrt erhalten.

Der zweite Doppeltrimeter ist zweimal stark interpoliert worden.

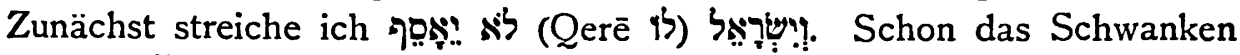
in der Überlieferung, welches in den ganz gleichartigen und ebenfalls interpolierten letzten drei Worten von v. 6 a wiederkehrt, kann zugunsten meiner Annahme angefüht werden; es kann auf undeutliche oder-schwankende Schreibung am Rande hinweisen. Verdächtig sind die drei Worte schon längst erschienen, da sie in die infinitivische Konstruktion des Satzes hineinstolpern. Liest man ל in der Glosse, so kann man das fragend auffassen, „und Israel sollte nicht (auch) versammelt werden?" Der Schreiber hätte dann den Gegensatz von Israel und Jakob in dem Gedichte nicht begriffen und wunderte sich, $\mathrm{da}$ bon Israel bei der Zurückführung gar nicht mehr die Rede ist. Liest man ib, so ist der Sinn derselbe, ,und (auch) Israel wird zu ihm versammelt werden". Ich vermute, daß der Glossator in der Tat das Gedicht in dieser Weise mißverstanden hat. Denkbar wäre es ja freilich auch, $\mathrm{da} \emptyset$ er richtig verstehend hätte sagen wollen ,und Israel wird nicht versammelt werden", vielleicht als Korrektur zu der Glosse v. 6ar; aber wenig wahrscheinlich. - Außerdem sind die drei letzten

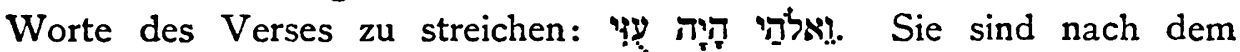
Muster von v. $4 \mathrm{~b}$ zugesetzt worden: Dort war nach genannt worden; daher glaubte ein Leser auch hier hinter nịi! noch

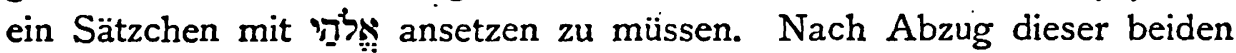
Glossen erscheint der zweite Doppeltrimeter des masoretischen Verses.

Metrische Gründe würden in v. 5 . nicht gegen die beiden Glossen sprechen; der masoretische Vers würde bei Beibehaltung beider Glossen drei Doppeltrimeter enthalten. Wohl aber sprechen in v. 6 a auch metrische Gründe für Ausscheidung der Israel-Glosse, und das fällt dann auch für v. 5 mit ins Gewicht.

An den Vorausblick „und ich bin wertgehalten worden vor Jahwe" schließt sich nun gleich v. 6 an, der die Verherrlichung Jahwes durch Israel, nämlich die Ausbreitung seines Kultus durch Israel verheibt. An Stelle des vielbesprochenen im hebräischen Text biș zu lesen. Für I hat der Schreiber I verschrieben, und 17 sind $z u \quad p$ geworden. "Größer als mir Knecht zu sein, um die Stämme Jakobs aufzurichten, habe ich dich gesetzt, zum Lichte der Heiden zu sein bis zum Ende der Erde." Das einleitende gehört $z \mathrm{u}$ den Ausschaltungen SiEvers' (Metrische Studien, \241, I); 
darauf folgt ein Doppeltrimeter bis zu בp:ir. Dann die Interpolation

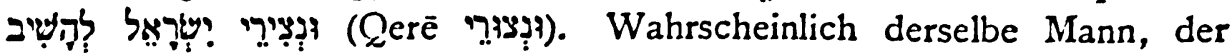
glaubte in v. $5 \mathrm{zu}$ Jakob auch noch Israel zufügen zu müssen, hat das


I man vielleicht streichen kann. 'יש:? zu streichen, ist des Metrums wegen nötig; das Wort wird von jemandem zugefügt sein, dem das etwas leer scheinende

In dem kurzen Zusatz v. 7 ist der fehlerhafte Konsonantentext לבוהדנפשי wals aktives

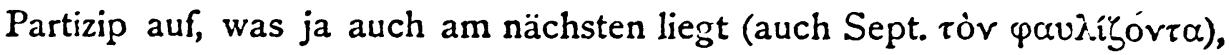
„zu dem Verächter einer Seele“. Diesen eigentümlichen Ausdruck er-


dachte bei ' $\Psi$, נֶֶֶ an die Babylonier. Ich glaube also nicht, dab die jetzt beliebte passivische Deutung von מתעב der Absicht des Schrei-

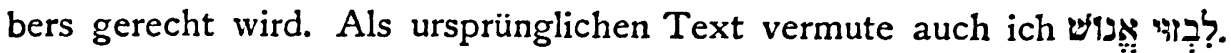
Das eigentliche Jahwewort, das mit מְלִ מִבְים beginnt, scheint in zwei Doppeltrimetern gestaltet $\mathrm{zu}$ sein. Am Schlusse des ersteren, d. h. hinter werden $z u$ müssen.

III.

Der erste Vers des dritten Gedichtes, 50 4, ist m. E. bisher mißverstanden worden. Von Jüngerzungen und Jüngern steht wohl nichts im Texte, wenigstens nicht im ursprünglichen Texte; und alles, was man daran angeknüpft hat, dürfte aufzugeben sein.

Von den לִ ist nur in v. 4 die Rede, nachher nicht mehr.

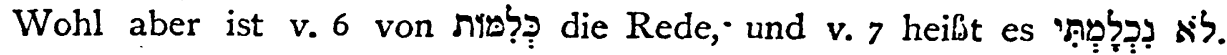
Ich zweifle nicht, daß auch am Schlusse von v. 4 כְְִּ zu lesen ist

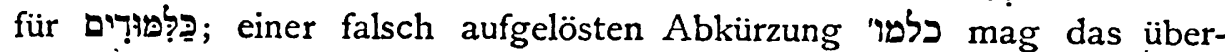
lieferte

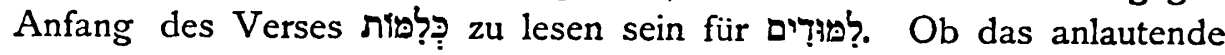
$\supset$ hier absichtlich oder unabsichtlich, etwa durch Verblassung der Tinte, aufgegeben worden ist, wird sich nicht entscheiden lassen. Erwägt man weiter, daß in der alten Schrift $\Delta$ und $ש$ zum Verwechseln einander ähnlich sehn, so erscheint das im überlieferten Text unmittelbar

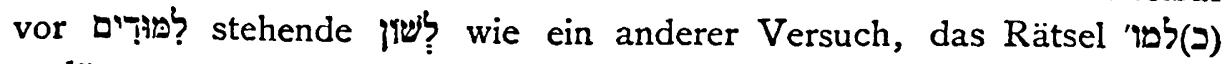
zu lösen.

Als ursprüngliche Gestalt von v. 4 a $\alpha$ nehme ich also an: ידוה נָתַן Jahwe hat'mir Schande gegeben." Aus v. 4 a $\beta$ läßt sich 


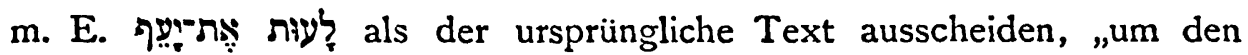
Schwachen (d. i. $=$ mich) zu demütigen". Vielleicht ist ת!? ک? zu lesen; für den Sinn wäre alles gleich. Ebenso auch für das Versmaß, den gewöhnlichen Pentameter. Die beiden Worte, welche jetzt außerdem noch in v. 4 a stehen, hat ein gründlicher Schulmeister dem bereits verunstalteten Texte beigeschrieben, um klar zu machen, daß die Zunge zum Reden (דבר) ist, daß die Schüler kennen lernen (לדעת) sollen.

V. 4 b lautet nach Streichung der Dublette am Anfang: עי:

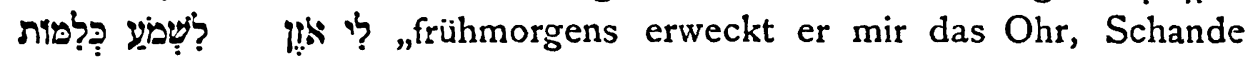
zu hören." Das ist ebenfalls ein regelrechter Pentameter.

In v. 5 a liegt ein weiterer Pentameter vor; nur der Gottesnamen am Anfang ist sekundär verdoppelt worden. Der Akzent liegt hier auf dem Gottesnamen; somit haben wir hier nicht eine bloße Variante zu v. $4 b^{2}$. V. 5 b ist zu streichen als erklärender Zusatz zu מִרִִ

- In v. 6 liegen zwei Pentameter vor, die im wesentlichen unversehrt erhalten sind. Das gleiche ist betr. v. 7 zu bemerken.

Aber v. 8 ist mit erklärenden Glossen stark überladen worden. Es


wer mit mir streitet." Dieser Satz war seinem Sinne nach nicht ganz klar; "ي̣ konnte aufgefaßt werden als „für mich" oder als "gegen mich". Deshalb erläuterte jemand den subjektischen Teil des Satzes durch

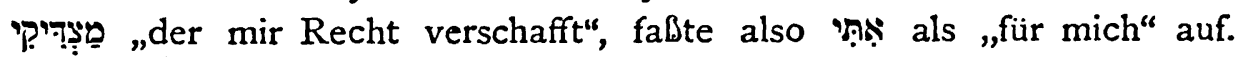
Weiter sollte der hier immerhin etwas ungewöhnliche relativische Ge-

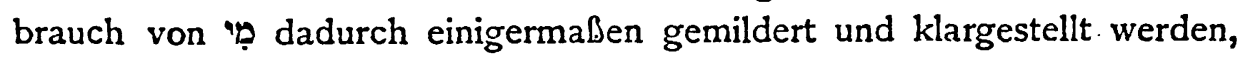
daß die Phrase unter Beibehaltung von leicht umgegossen wurde: „Wer mein Rechtsanwalt, er nähert sich mir." In dieser Umgießung ist

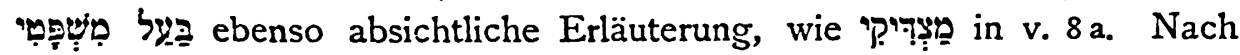
Ausscheidung dieser Glossen bleibt ein richtiger Pentameter übrig.

In v. 9 wieder zwei anscheinend unversehrte Pentameter.

Durch Ausschaltung der Zungen und der Jünger verläuft das ganze Stück einheitlich und verständlich: Der Knecht ist in Schande und Not und erträgt alles ohne zu murren und ohne sich seinem Schicksal zu widersetzen. Aber Jahwe wird ihm helfen, und seine Gegner werden schwinden.

Das angehängte kleine Stück v. 1o und II scheint eine Nutzanwendung sein $\mathrm{zu}$ wollen. Haec fabula docet. Macht es ebenso wie der Knecht, wenn es euch schlecht geht; vertrauet auf Jahwe! Aber das tut ihr ja doch nicht! 
Das Stück läuft in fünf Doppeltrimetern. Der erste dieser Doppeltrimeter ist wohl unversehrt erhalten, abgesehn davon, dal (mit Sept.)

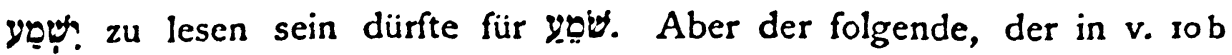
vorliegt, ist stark erweitert worden: "Wer wandelt in dem ihm kein Lichtschein ist" ist durch

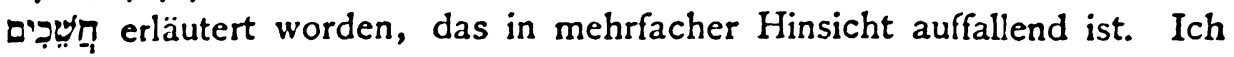
vermute, es wird Rest sein von den Namen Jahwes." Hier schien der Name Jahwes jemandem etwas $z u$ eng zu sein; daher veränderte er den Ausdruck nach der allgemeinen Richtung hin: „Der möge sich stützen auf seinen Gott" (oder Sept. ė $\pi i$ $\tau \bar{\varphi} \vartheta \vartheta(\tilde{)})$.

Viele Schwierigkeiten macht v. Ir. Ja, wenn wir wübten, was תip'!


pfeile entzündet" ist schon metrisch verdächtig, weil die so veränderten Worte nicht gut die hier verlangten drei Ikten tragen können. Wohl

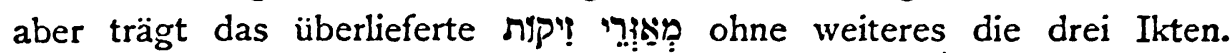
Und welcher Sinn! - Ich vermute: "Siehe, ihr alle seid Feuer anzündend, mit (Wasser)schläuchen angetan; ihr geht in der Flamme eures Feuers und habt (sie) mit den Schläuchen in Brand gesetzt." Der Sinn wäre also etwa: „Ihr fangt mit den guten Vorsätzen zwar an, hört aber bald wieder auf." „Mit Schläuchen umgürtet." Man denke an bekannte Abbildungen der morgenländischen Saqqās, mit dem Wasserschlauch auf dem Rücken und an der Seite. Für לִ: vielleicht

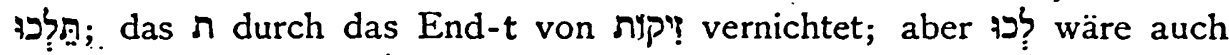
möglich. Für änderung wäre nicht groß; und ein Schreiber konnte leicht zu der Veränderung in בערתם verführt werden, $\mathrm{da}^{*}$ so verführerische Worte wie

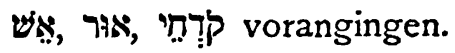

\section{IV.}

Das vierte Gedicht beginnt 5213 „siehe Einsicht haben wird mein

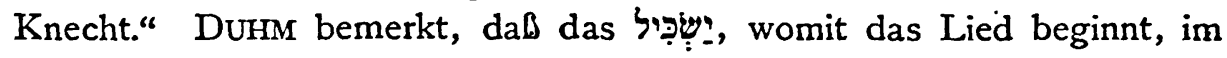
weiteren Verlaufe desselben zu keiner rechten Geltung komme und nur an 53 II eine schwache und zweifelhafte Stuitze habe; er streicht daher :- Das scheint mir nicht richtig zu sein. So viel Dunkles. das Gedicht auch bietet, so viel scheint mir sicher zu sein, daß Inhalt und Ziel des Gedichtes grade darauf hinausgehen, daß der von Gott berufene Knecht durch sein Leiden und Sterben (?) „Einsicht" erwirbt. Aber was hier unter "Einsicht" zu verstehen ist, darüber wissen wir 
nichts Bestimmtes. Man deutet das betreffende Wort hier gern nach der Richtung „Erfolg“ hin. Natürlich nur als Vermutung möchte ich aussprechen, dab unter „Einsicht" hier vielleicht ein besonderer Grad der Gotterkenntnis verstanden sein könnte, von der im AT so oft die Rede ist (auch wohl im vierten Gedicht 53 II), etwas dem ähnliches, was man anderswo $\gamma v \tilde{\omega} \sigma ı \zeta$ genannt hat.

Grade am Schlusse des Gedichtes, 53 II 12, kommt der Dichter wieder darauf zurück. Der Text ist freilich nicht mehr unversehrt erhalten, läßt sich aber mit einiger Sicherheit wiederherstellen.

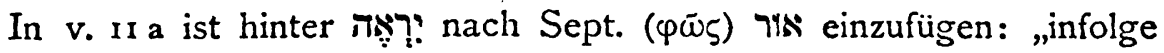
der Mühsal seiner Seele wird er das Licht schauen." Aus REITZENSTEIN, Die hellenistischen Mysterienreligionen, S. I I9 ersehe ich, daß

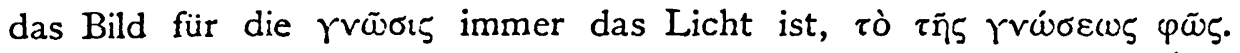
$\mathrm{Daß}$ der hebräische Dichter hier רז gebraucht habe, wage ich nun allerdings nicht zu behaupten im Hinblick auf SEllin, Studien, I. Bd., S. 95 f.; Rätsel S. 73, so nahe diese Vermutung hier auch liegen mag. Also mag Tis hier immerhin etwa „Heil“ bedeuten; im folgenden ist deutlich vom Erkennen und von „Einsicht" die Rede.

"Gesättigt wird er werden durch sein Erkennen (und) gerecht-

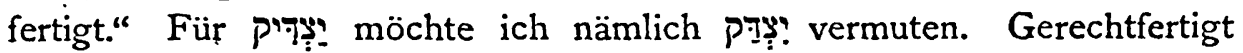
werden ist hier wohl gesagt in besonderem Hinweis darauf, daß der Knecht früher zu den Übeltätern gezählt worden war; nicht in dem allgemeinen Sinne, daß überhaupt jeder mit „Einsicht“ begnadete dadurch sündlos werde. Mit diesem Pדঙ:? endet der erste Doppeltrimeter des masoretischen Verses; der zweite läuft von pִִ̣ bis zum Schlusse. „Gerechtfertigt ist mein Knecht für viele, und ihre Sünden trägt er." Das soll doch wohl heißen, daß nicht nur der Knecht selber gerechtfertigt ist, sondern auch die vielen, denen er die Sünden abgenommen hat. Aber die Ausdrucksweise ist nicht ganz klar; und es scheint mir, als habe das nicht zum ursprünglichen Text gehörige in v. 12 a ursprünglich als Glosse zu לָרבְבִ am Rande gestanden ,gerechtfertigt ist mein Knecht mit vielen".

V. 12 a ist zu Anfang nicht nur völlig unmetrisch, sondern auch unverständlich, mindestens stark ineinander geschachtelt. Grund genug, dem überlieferten Texte zu mibtrauen. Sept. hat $\kappa \lambda \eta p o v o u n ́ \sigma \varepsilon \imath$ statt

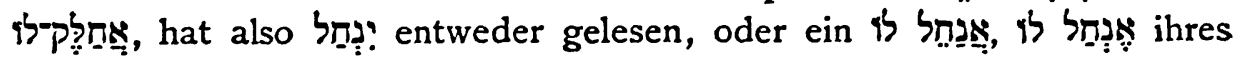
Textes sich in die dritte Person zurechtgelegt (vgl. DuHM z. St.). Ich

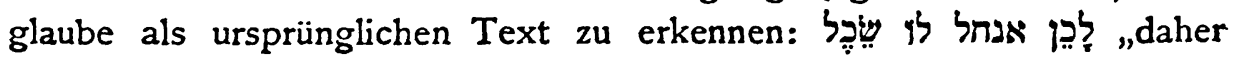


werde ich ihm Einsicht zuerteilen." Das ist die erste Hälfte des erwarteten Doppeltrimeters. שֵֶֶׁ wurde in verschrieben, und in der Folge wurde אנח in das bedeutungsverwandte, mit fast denselben Buchstaben geschriebene zu werden pflegt. Sept. hat in ihrem Texte bereits gefunden, daneben aber noch das ursprüngliche s אל Von Krieg, Beute und dergleichen ist sonst nirgends die Rede, auch in den drei anderen Gedichten nicht. Man hat daher, bei dem überlieferten Texte stehen bleibend, entweder annehmen müssen, dab hier tatsächlich eine Verheißung an den in Zukunft siegreichen König-Knecht vorliegt (so Sellin, Rätsel, S. 75), oder man hat den Begriff Beute verallgemeinern und umbiegen müssen.

Was zwischen is und (שֶֶֶל steht, ist späterer Zusatz, an-

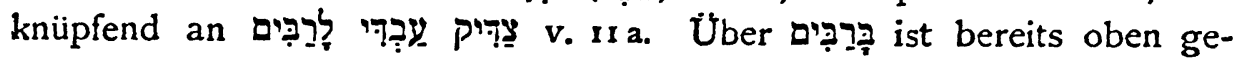
sprochen worden. Da nun der Knecht "Beute" erhalten soll, schien die Folge zu sein, daß auch "die vielen", für welche oder mit welchen er.gerechtfertigt ist, ebenfalls Beute erhalten müßten. Daher schrieb

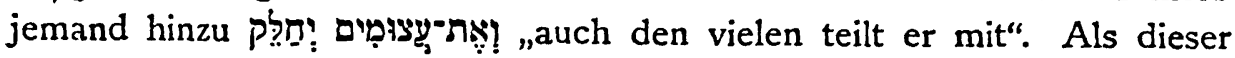
Glossenschreiber schrieb, stand bereits שָ im Texte; daher schrieb er

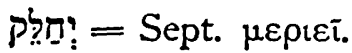

Die zweite Hälfte des Doppeltrimeters scheint unversehrt vorzuliegen

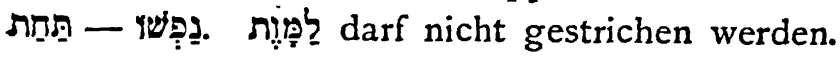

Der letzte Doppeltrimeter des Gedichtes scheint ebenfalls unversehrt erhalten zu sein. Nur die beiden letzten Worte

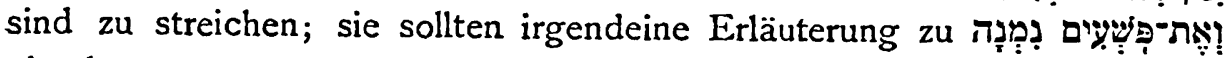
abgeben.

Nach dieser Deutung der beiden letzten Verse des Gedichtes werden wir das 'שְ:- am Anfange beibehalten müssen. An reihen sich in v. I3b noch drei andere verbale Prädikate. $D a ß$ von ihnen eins zu streichen ist, wird durch die Metrik verlangt; auch Sept. hat hier nur zwei Verba. Ich streiche zuversichtlich is das ich als erklärende Glosse zu dem mehrdeutigen נשא auffasse. Dann liegt in v. 13 der erwartete Doppeltrimeter vor.

Die beiden angereihten Prädikate „er wird erhaben sein und sehr erhöht werden" führen wohl auf ein anderes Gebiet. Da v. 15 den Knecht viele Völker preisen (?) werden, die Könige (vor Bewunderung, Ehrfurcht) den Mund zuhalten werden, scheint es doch näherliegend, an irdische Hoheit und an irdische Ehren zu denken, als an eine örtliche Erhöhung zu Gott, um ihn zu schauen und zu erkennen. 
Das doppelte folgender Sätze hat schon die alten jüdischen Leser gestört. Daher hat einer den Inhalt des ersteren durch פִ eingeleiteten Satzes durch

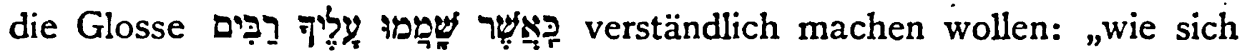
viele über dich entsetzen, so ..." רִִִִָים in dieser Glosse ist aus dem zweiten kēn-Satze hergeholt. Fיר ist ungenau, aber in der Glosse begreiflich; vielleicht war das ebenfalls nicht zum ursprünglichen Texte

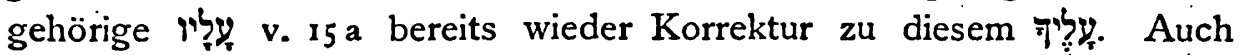
metrisch wäre die Glosse als Hälfte eines Doppeltrimeters zu lang, außerdem würde man die andere Hälfte vergeblich suchen. Streicht man aber diese, an die Spitze des masoretischen Verses gestelite Glosse, so bleib't der erwartete Doppeltrimeter übrig.

Ich glaube, daß wir in - רֶּ - allerdings eine ungewöhnliche Ausdrucksweise $\mathrm{zu}$ sehen haben: so - , so $-=$ wie - , so - ; und glaube, daß der alte Glossator das Richtige getroffen hat, wenn er das erstere in in iַ umsetzte.

In v. 15 a möchte ich für das vielbesprochene i!: vorschlagen „So werden viele Völker lobpreisen“. Man wird die Ähnlichkeit der Schriftzüge bemerken. $\mathrm{Da}$ " י es ist metrisch unmöglich. Im übrigen sind in v. 15 zwei unversehrte Doppeltrimeter erhalten.

$53 \mathrm{Ib}$ ist metrisch überfüllt; und wie so oft ist auch hier der

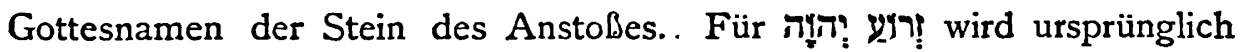
Iעוฺ im Text gestanden haben. Dann haben wir in 53 I einen regelmäßigen Doppeltrimeter. $\mathrm{Daß}$ das Pronominalsuffix erst später durch das Substantiv ersetzt worden ist, darauf haben wir außer dem metrischen Befund noch einen anderen Hinweis. In Sept. nämlich heißt es

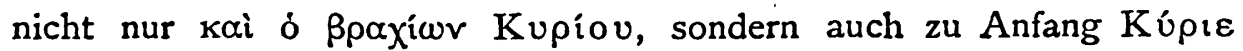

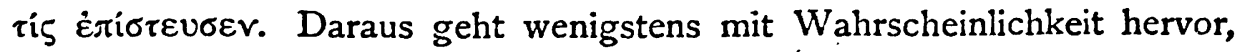
dab יהוה zuerst als Glosse zu ị an den Rand geschrieben worden

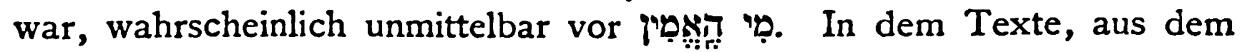
Sept. übersetzten, wurde יהוה dann nicht bloß an Stelle des Pronominalsuffixes in die Mitte des Textes eingerückt, sondern behauptete sich auch am Anfang.

532 , "und er stieg auf" pabt schlecht in den Zusammenhang, in dem von Niedrigkeit und Elend die Rede ist; und auch durch die übliche Umbiegung in die Bedeutung "und er wuchs auf" entsteht ein recht matter und zweifelhafter Sinn, selbst wenn man, wie üblich, pয়r



Zeitschr. \&. d. alttest. Wiss. Jahrg. 36. 1916. 
dürfte der ursprüngliche hebräische Text anders gelautet haben. Ich vermute und wie eine Wurzel aus dürrer Erde". Aus diesem Zusammenhange

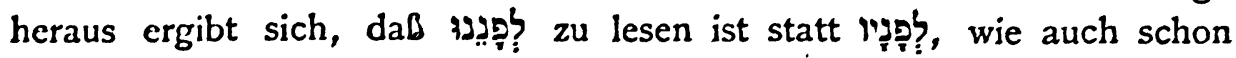
früher vermutet worden ist. - Sept. scheint gelesen zu haben.

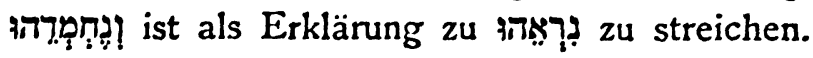

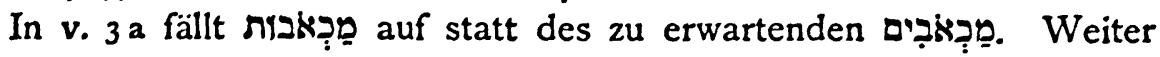
fällt auf die seltene Pluralform folgt. Diesem folgt nun wieder ein $\mathrm{m}$, das anlautende $\mathrm{m}$ des be-

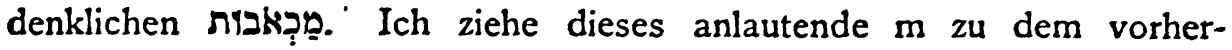
gehenden שִ̣, also ursprünglich als אנששים Erklärung des ersten sein sollte. Ich


,verachtet und ${ }^{2}$ aufgehört habend Mensch zu sein, wie





Es bleiben in v. 3 a aber noch die beiden letzten Worte zu erklären

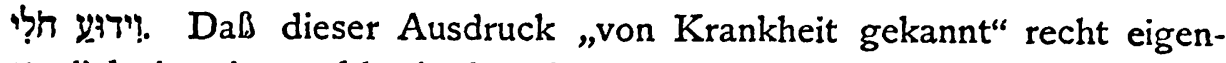
tümlich ist, ist wohl nie bestritten worden. Man erinnere sich nun, daß im AT mit den אביר zự gusammen immer die genant werden;

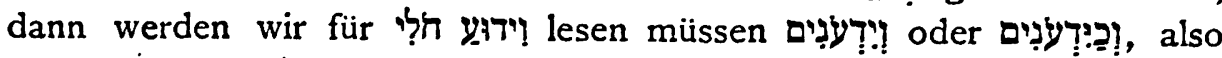
"wie Gespenster und Spuk“. Vielleicht hat man die Gespenster und den Spuk absichtlich aus dem Text entfernt, da es anstößig erscheinen konnte, den "Messias" mit solchen unreinen Geistern zu vergleichen; vielleicht gab aber die unbeabsichtigte falsche Worttrennung bei אישמכאבות den ersten Anstob zu der Verderbnis des Textes, der dann das weitere folgte.

Der v. 3 a bildet in seiner obigen Wiederherstellung einen richtigen Doppeltrimeter, während er in der überlieferten Gestalt unmetrisch ist. V. 3 b ist metrisch richtig und auch sonst wohl im ganzen unversehrt und verständlich überliefert. Erwägt man, daß unmittelbar vorher von Gespenstern und Spuk die Rede war, so liegt die Vermutung nah, daß "und wie einer vor dem man das Gesicht versteckt" diesen Gedanken fortsetzt, daß damit besonders diejenigen Geister gemeint sein sollen, bei deren Erscheinen man die Augen abwenden muß. Vgl. W. KROLL in Mitteilungen der Schlesischen Gesellsch. f. Volkskunde, Bd. 13/14, S. 480 .








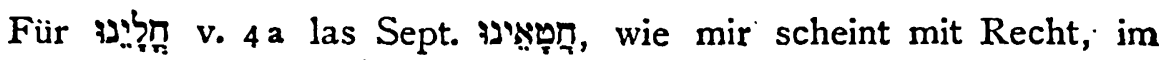


wesen zu sein. Auch in v. 3 a ist ${ }^{\text {. }}$ irrtümlich in den ursprünglichen Text hineingetragen worden, ebenso in v. roa.

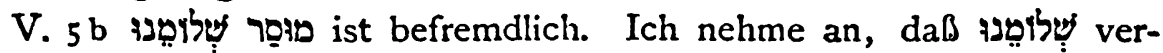

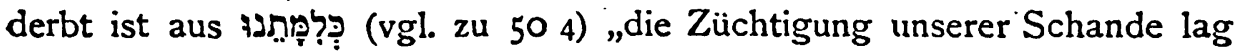
auf ihm“. $\supset$ und $y$ sind in der alten Schrift leicht zu verwechseln; vgl. Jahrg. I9I5, S. $14 \mathrm{zu}$ Am 44 b. Ich hatte diese Veränderung bereits vorgenommen, ehe ich gesehen hatte, daß Hi 203 tatsächlich die



V. $6 \mathrm{~b}$ ist durch seine metrische Form auffallend, und ich glaubte zuerst, hierin einen Hinweis auf Unechtheit sehen zu müssen. Statt des erwarteten Doppeltrimeters finden wir nämlich in v. 6b einen Doppelbimeter. Doch dürte das nur scheinbar und äußerlich sein; vielmehr dürfte dieser scheinbare Doppelbimeter rhythmisch als Doppeltrimeter zu bewerten sein. Der Dichter hat vor der Cäsur und am Versschlusse parallele Pausen eingesetzt, dadurch das Gewaltige des Gegensatzes hervorhebend:

$$
\text { weyahwé hifgîi bó } \smile-\text { et awớn kullánu - }
$$

In v. 7 a ist נגש


ja auch festgehalten worden ist. Streicht man diese Glosse, so erscheint der mit לָּ" schließende erste Doppeltrimeter des masoretischen Verses.

In dem schwierigen achten Verse ist seinem Wortlaute nach sicher vom Tode des Knechtes die Rede. Ob dieser Tod in des Wortes eigentlicher Bedeutung zu nehmen ist, oder nur als Bild für Not und Unglück, das muß ich unentschieden lassen. $\mathrm{Da}$ in dem Verse die erwarteten beiden Doppeltrimeter in metrischer Hinsicht ziemlich anstoblos vorliegen, so dürfen wir vielleicht hoffen, dab der Vers in seiner textlichen Überlieferung überhaupt besser ist, als man bei der Schwierigkeit seines Verständnisses annehmen könnte.

„Aus Gefängnis und Strafgericht wurde er weggenommen, und wer sinnt nach über seinen Tod?“ Für das vielerörterte nämlich is lesen. Nicht nur aus den allgemeinen Gründen, daß מת gut in den Zusammenhang passen würde, und daß auch seine Schriftzüge sich von 17 ग nicht weit entfernen, sondern auch noch aus einem besonderen Grunde: Am Schlusse des Verses steht 4 i ל̧, abgekürzt aus תוּ, wie Sept. noch las. Dab ein Schreiber abgekürzt למו schrieb, 
war jedenfalls dann weit näherliegend, wenn dasselbe Wort bereits kurz vorherging, als wenn es zum ersten Male in der Erzählung auftrat.

Den v. 9 a möchte ich verstehen: „Und er nahm sich bei den Übeltätern sein Grab und bei den Wüstendämonen seine Wohnung“, indem


Verses scheinen in einer Handschrift aus Raummangel etwas überein. ander und durcheinander geschrieben gewesen $z \mathrm{u}$ sein, so dab die jetzige unverständliche Lesart entstehen konnte. Und was den Sinn der Worte betrifft, so scheinen sie bedeuten $z u$ sollen, daß der sündenbeladene Knecht schon bei Lebzeiten in die Wüste gejagt worden ist, wie der Sündenbock, und dab auch sein Grab bei den sündenbeladenen Menschen gegraben wurde. Vgl. noch WúNSCH in Mitteilungen der Schles. Gesellschaft für Volkskunde, Bd. 13/14, S. 17f. Auch hier wären die unreinen Geister aus dem Texte entfernt worden, wie v. 3 a In v. 10 a gehört החלי nicht dem ursprünglichen Texte an. Der, welcher das Wort zuerst zufügte, wollte das Suffix von i⿱彐 İ erklären "Jahwe wünschte ihn zu zermalmen, d. h. die Krankheit.“ Vielleicht

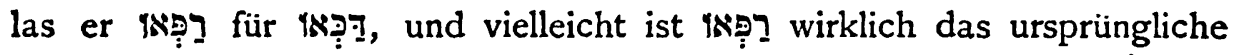



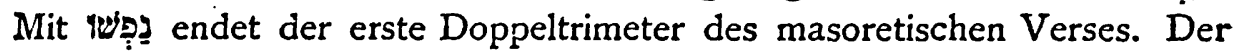
2weite ist in dem Reste enthalten; nur ist für den Gottesnamen wieder das Suffix einzusetzen: 